\title{
PCA-Combined Receive Antenna Selection for MMSE V-BLAST in Correlated Channels
}

\author{
Chia-Chang Hu and Chi-Tsung Wang \\ Department of Communications Engineering, National Chung Cheng University, Min-Hsiung, Chiayi 621, Taiwan \\ Correspondence should be addressed to Chia-Chang Hu, ieecch@ccu.edu.tw
}

Received 9 December 2010; Accepted 18 January 2011

Academic Editor: E. Ciaccio

Copyright (C) 2011 C.-C. Hu and C.-T. Wang. This is an open access article distributed under the Creative Commons Attribution License, which permits unrestricted use, distribution, and reproduction in any medium, provided the original work is properly cited.

A computationally efficient receive antenna subset selection in conjunction with the principal component analysis (PCA) is proposed for the minimum mean square error (MMSE) V-BLAST systems in correlated channels. This proposed PCA-combined antenna subset selection is capable of sustaining system performance and reducing complexity burden in signal processing and hardware cost.

\section{Introduction}

The major limitation on the deployment of a V-BLAST system is the cost, complexity, and power consumption of multiple complete radio frequency (RF) chains associated with the multiple antennas [1]. To mitigate this problem, multiple-input multiple-output (MIMO) links with making full use of antenna subset selection have been proposed in $[1,2]$. These antenna selection schemes reduce the hardware complexity of transmitters and receivers by using fewer RF chains than the actual number of antenna elements. Another drawback incurred by the utilization of multielement antennas (MEA) at both sides in a V-BLAST system is the large dimension of the channel matrix. To overcome the heavy burden in signal processing, the reduced-rank approach is applied to perform signal compressing prior to signal processing. The principal component analysis (PCA) [3] is one of the widely used techniques for the reducedrank signal processing. In this paper, the strengths of both the antenna subset selection and the PCA algorithm are combined together to offer a distinguished and speedy sequential signal detection with reduced system hardware and signal processing for MMSE-based V-BLAST systems in correlated channels. The following notation is used: symbols for matrices (vectors) are denoted by boldface upper (lower) case letters. The superscript $(\cdot)^{\mathrm{H}}$ stands for
Hermitian transposition. $E\{\cdot\}$ denotes the expected-value operator. $\operatorname{det}(\cdot)$ gives the determinant of a matrix. $\mathbf{I}_{M}$ is an $M \times M$ identity matrix. $|\cdot|$ represents the absolute value. $(\mathbf{H})_{:, i}$ indicates the $i$ th column vector in the matrix $\mathbf{H}$. Finally, $Q(\cdot)$ is the $Q$ function.

\section{System Model}

In the V-BLAST MIMO transceiver, the numbers of the transmitting and receiving antennas are $N$ and $M$, respectively. The selection circuit selects $L$ antennas out of the total of $M$ available receive antennas based on antenna selection criteria. Subsequently, the PCA technique is applied to reduce the rank of the resulting channel matrix to mitigate signal processing complexity while maintaining system error performance. The channel model is assumed to be flat fading and memoryless, then the input-output relationship of a MIMO system in matrix form can be described by

$$
\mathbf{r}=\sqrt{\frac{\rho}{N}} \mathbf{H} \mathbf{x}+\mathbf{n}
$$

where $\mathbf{r}$ is the $M$-dimensional received signal vector, $\mathbf{x}$ is the $N$-dimensional transmitted signal vector with equally distributed transmit power (i.e., $E\left\{\mathbf{x x}^{\mathrm{H}}\right\}=\mathbf{I}_{N}$ ), $\mathbf{n}$ is the $M$-dimensional independent and identically distributed (IID) zero-mean complex additive white Gaussian noise 
(AWGN) vector with energy $N_{0} / 2$ per complex dimension, $\rho$ corresponds to the average signal to noise ratio (SNR) at each receive antenna, and $\mathbf{H}$ is an $M \times N$ random channel matrix. The capacity of the MIMO channel $\mathbf{H}$ is presented by [1]

$$
\begin{aligned}
\mathrm{C}(\mathbf{H}) & =\log _{2}\left[\operatorname{det}\left(\mathbf{I}_{M}+\frac{\rho}{N} \mathbf{H} \mathbf{H}^{\mathrm{H}}\right)\right] \\
& =\log _{2}\left[\operatorname{det}\left(\mathbf{I}_{N}+\frac{1}{N_{0}} \mathbf{H}^{\mathrm{H}} \mathbf{H}\right)\right] .
\end{aligned}
$$

To reflect the effect of spatial correlation, a correlated Kronecker channel matrix [4] written as $\widetilde{\mathbf{H}}=\mathbf{R}_{r}^{1 / 2} \mathbf{H}_{\omega} \mathbf{R}_{t}^{1 / 2}$ is employed, where $\mathbf{H}_{\omega} \sim C N(0,1)$ represents a stochastic $M \times N$ matrix with IID complex Gaussian zero-mean, unitvariance entries. Matrices $\mathbf{R}_{r}$ and $\mathbf{R}_{t}$ denote, respectively, the $M \times M$ and the $N \times N$ antenna correlation matrices at the receiver and the transmitter. The approximation of the spatial cross-correlation function $r(d)$, that determines the correlation between two adjacent antenna elements separated in space by a distance $d$ [5], is given as follows: $r(d) \approx \exp \left[-23 \mathrm{~A}^{2}(d / \lambda)^{2}\right]$, where $\lambda$ is the wavelength and $\mathrm{A}$ is the angular spread parameter, which is defined by $\mathrm{A}=$ $\sqrt{\theta^{2}+2 \cos \theta-2} / 2 \theta$, where $\theta \in[0,2 \pi]$ denotes the width of the sector of arriving multipath power.

\section{Receive Antenna Selection Criteria}

To reduce the need for multiple expensive RF chains at both ends yet retain diversity merits, two categories of capacityand performance-based antenna subset selection schemes are utilized as follows:

3.1. Capacity-Based Antenna Subset Selection. With the use of (3), the capacity of the MIMO channel $\tilde{\mathbf{H}}$ can be reformulated as [6]

$$
\begin{aligned}
\mathrm{C}(\tilde{\mathbf{H}}) & =-\log _{2}\left[\operatorname{det}\left(\mathbf{I}_{N}+\frac{1}{N_{0}} \widetilde{\mathbf{H}}^{\mathrm{H}} \tilde{\mathbf{H}}\right)^{-1}\right], \\
& =-\log _{2}\left(\prod_{k_{i}=1}^{N} \mathrm{MSE}_{k_{i}}\right),
\end{aligned}
$$

where $\mathrm{MSE}_{k_{i}}=N_{0}\left[\left(\overline{\widetilde{\mathbf{H}}}_{k_{i}}^{\mathrm{H}} \overline{\widetilde{\mathbf{H}}}_{k_{i}}+N_{0} \mathbf{I}_{N}\right)^{-1}\right]_{k_{i} k_{i}}$ defines the MSE for the $k_{i}$ th detection stage in the MMSE V-BLAST system and the matrix $\overline{\widetilde{\mathbf{H}}}_{k_{i}}$ is obtained by setting all the $k_{1}$ th, $k_{2}$ th,..., $k_{i-1}$ th columns of $\tilde{\mathbf{H}}$ to zeros. Thus, according to (4), to maximize the capacity of the MMSE V-BLAST system based on the selected antenna subset, $c_{i}$ is equivalent to minimize the products of MSEs at all stages for $i=1,2, \ldots,\left(\begin{array}{c}M \\ L\end{array}\right)$.

3.2. Performance-Based Antenna Subset Selection. An upper bound of the MMSE V-BLAST receiver is derived as [7]

$$
P \leq N K Q\left(\sqrt{\operatorname{SINR}_{\min } \cdot \frac{d_{\min }^{2}}{2}}\right),
$$

where $P$ is the probability of the vector symbol error with at least one detected symbol error, $K$ is the number of nearest neighbors in the transmit signal constellation, SINR $_{\min }$ is the minimum postprocessing SINR among all the substreams, and $d_{\min }^{2}$ is the squared minimum Euclidean distance between any two symbols in the transmit signal constellation. Under the assumption that the energies of the transmitted symbols are normalized, (5) can be re-expressed as

$$
P \leq N K Q\left(\sqrt{\mathrm{MSE}_{\max }^{-1} \cdot \frac{d_{\min }^{2}}{2}}\right) .
$$

According to (6), the antenna subset with the minimummaximum MSE (minmax MSE) over a complete of $N$ detection stages is selected. Therefore, the subset of antennas $c_{k}$ is selected over all possible antenna combinations when it satisfies $k=\arg \min _{i \in(\underset{L}{M})} \mathrm{MSE}_{\max , i}$, where $\mathrm{MSE}_{\max , i}$ denotes the maximum MSE for the antenna combination $c_{i}$.

\section{The PCA Algorithm}

To offer a good tradeoff between system diversity gain and signal processing complexity in a V-BLAST system, the combination of the antenna selection and the PCA is considered. After performing the antenna selection, the PCA algorithm is applied to the channel matrix $\widetilde{\widetilde{\mathbf{H}}}_{c_{k}}$, which is relevant to the selected antenna subset $c_{k}$, as follows:

$$
\overline{\widetilde{\mathbf{H}}}_{c_{k}}^{S}=\mathbf{W}_{S}^{\mathrm{H}} \overline{\widetilde{\mathbf{H}}}_{c_{k}}
$$

where $\widetilde{\widetilde{\mathbf{H}}}_{c_{k}}$ and $\mathbf{W}_{s}$ in (7) denote the $S \times N$ and $M \times S$ matrices, respectively. The matrix $\mathbf{W}_{s}$ is formulated by the singular vectors denoted by $(\mathbf{W})_{:, i}$, for $i=1, \ldots, S$, which are associated with the largest $S$ singular values of $\widetilde{\widetilde{\mathbf{H}}}_{c_{k}}$. Note that those singular vectors $(\mathbf{W})_{:, i}$ can be tracked by means of the PASTd algorithm [8] with a substantially-reduced complexity. Finally, the fast recursive MMSE-based V-BLAST detection algorithm proposed in [9] is employed to further reduce the complexity load in calculating an ordered set of nulling vectors from the resulting channel matrix $\overline{\widetilde{\mathbf{H}}}_{c_{k}}$.

\section{Numerical Results}

A Kronecker channel model [4] with the use of $d_{t}=d_{r}=\lambda / 2$, $\theta_{t}=\theta_{r}=4 \pi / 9$ is considered. Here, $d_{t}$ and $d_{r}$ denote, respectively, the equidistant antenna interelement spacings of uniform linear arrays (ULAs) at the transmitter and the receiver. The angles $\theta_{t}$ and $\theta_{r}$ in radians indicate the distribution angles of arriving multipath power with respect to the broadsides of both the transmitter and the receiver antenna arrays. Additionally, the 16-QAM modulation scheme is considered, and the fast recursive MMSE-based V-BLAST detection algorithm in [9] is employed in simulations. All experimental curves are obtained by means of performing $10^{5}$ independent trials and then calculating their system error rates.

Figure 1 compares the averaged symbol-error-rate (SER) performance of the MMSE V-BLAST system with fixed 


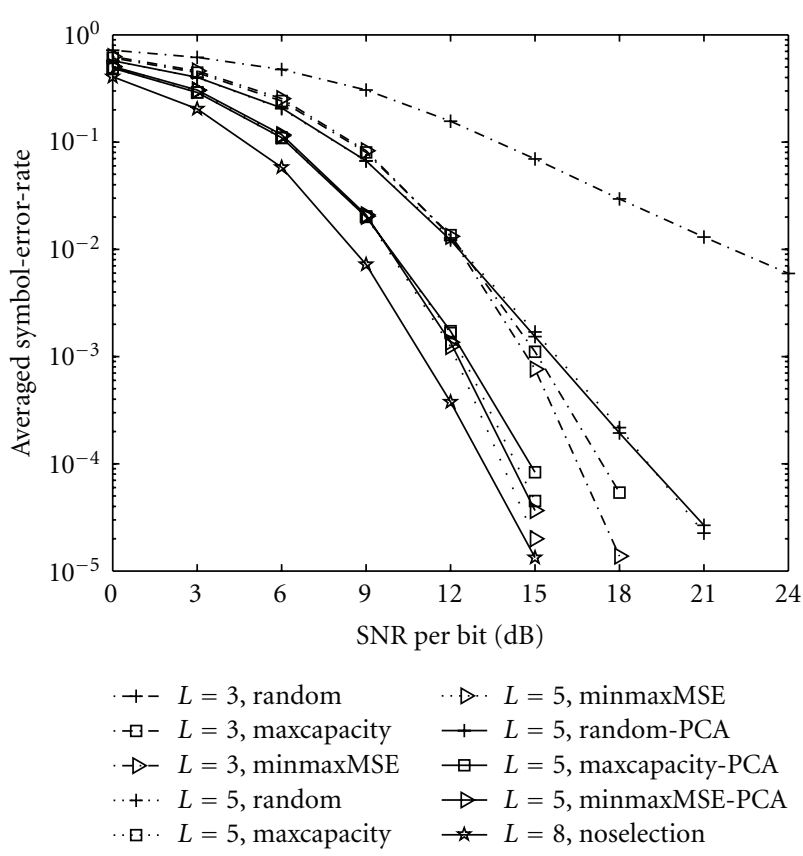

FIGURE 1: The averaged SER performance of the MMSE V-BLAST system with $(M, N)=(8,3)$ for various antenna selection criteria and $L$ RF chains at the receiver.

antenna configuration $(M, N)=(8,3)$ and $S=\min (M, N)$ in terms of SNR parameterized by various antenna selection criteria and $L$ available RF chains at the receiver. In the figure, the antenna subsets determined by the capacity- and the minmaxMSE-based criteria have the same asymptotic slope with the complete antenna set. This fact confirms that the use of the receive antenna selection is capable of providing the same diversity order as a full complexity (FC) wireless system with $M=8$ receive antenna elements and associated multiple RF chains. Moreover, it is also seen from Figure 1 that the 'minmaxMSE' method produces better SER performance than those of the "maxcapacity" and the "random" techniques no matter whether the PCA is employed or not. Notably, this fact implies that the corresponding antenna subset derived from the capacitybased selection criterion may not be the best antenna subset in terms of system error probability. In addition, with the use of the PCA, the system of " $L=5$, minmaxMSEPCA" outperforms the " $L=3$, minmaxMSE" one while maintaining the computational complexity order. Moreover, the system of " $L=5$, minmaxMSE-PCA" accomplishes nearly the same averaged SER performance as those of the " $L=8$, minmaxMSE" and the " $L=5$, minmaxMSE" systems but a much lower signal processing complexity owing to the adoption of the PCA algorithm. Furthermore, the "random" antenna selection provides a worst SER performance among those antenna selection techniques.

\section{Conclusions}

In this paper, a reduced-complexity PCA-combined receive antenna subset selection is proposed for MMSE V-BLAST systems over a correlated channel. From results, it is evident that a considerable reduction in system hardware cost and signal processing complexity is achieved by means of the receive antenna subset selection and the PCA scheme.

\section{References}

[1] S. Sanayei and A. Nosratinia, "Antenna selection in MIMO systems," IEEE Communications Magazine, vol. 42, no. 10, pp. 68-73, 2004.

[2] H. Zhang, A. F. Molisch, and J. Zhang, "Applying antenna selection in WLANs for achieving broadband multimedia communications," IEEE Transactions on Broadcasting, vol. 52, no. 4, pp. 475-482, 2006.

[3] I. T. Jolliffe, Principal Component Analysis, Springer, New York, NY, USA, 2004.

[4] D. S. Shiu, G. J. Foschini, M. J. Gans, and J. M. Kahn, "Fading correlation and its effect on the capacity of multielement antenna systems," IEEE Transactions on Communications, vol. 48, no. 3, pp. 502-513, 2000.

[5] G. D. Durgin and T. S. Rappaport, "Effects of multipath angular spread on the spatial cross-correlation of received voltage envelopes," in Proceedings of the 49th IEEE Vehicular Technology Conference (VTC '99), vol. 2, pp. 996-1000, Houston, TX, USA, May 1999.

[6] D. Lu and D. K. C. So, "Performance based receive antenna selection for V-BLAST systems," IEEE Transactions on Wireless Communications, vol. 8, no. 1, pp. 214-225, 2009.

[7] R. W. Heath Jr. and A. Paulraj, "Antenna selection for spatial multiplexing systems based on minimum error rate," in Proceedings of the International Conference on Communications (ICC '01), vol. 7, pp. 2276-2280, St. Petersburg, Russia, June 2001.

[8] B. Yang, "Projection approximation subspace tracking," IEEE Transactions on Signal Processing, vol. 43, no. 1, pp. 95-107, 1995.

[9] T. H. Liu and Y. L. Yeh Liu, "Modified fast recursive algorithm for efficient MMSE-SIC detection of the V-BLAST system," IEEE Transactions on Wireless Communications, vol. 7, no. 10, pp. 3713-3717, 2008. 

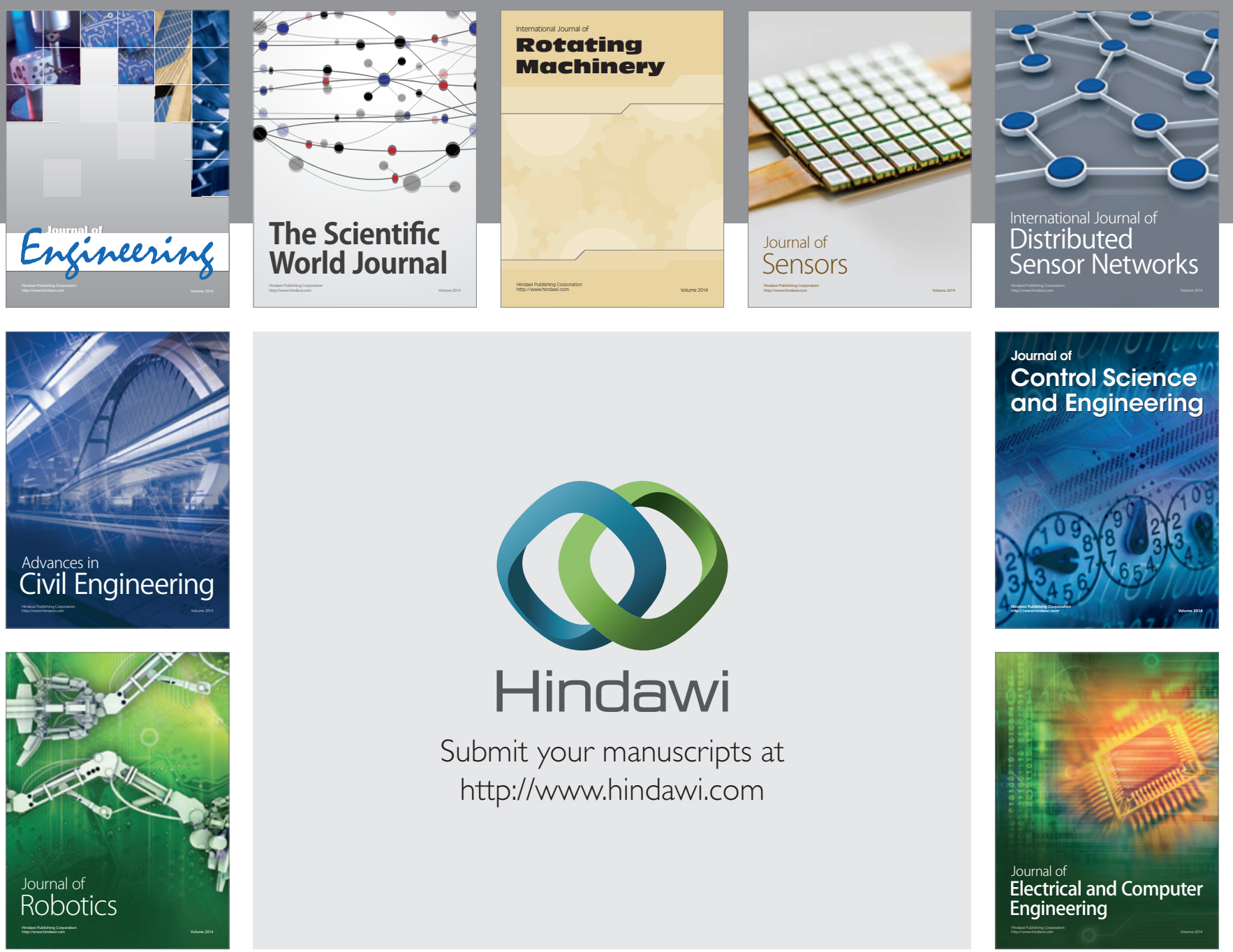

Submit your manuscripts at

http://www.hindawi.com
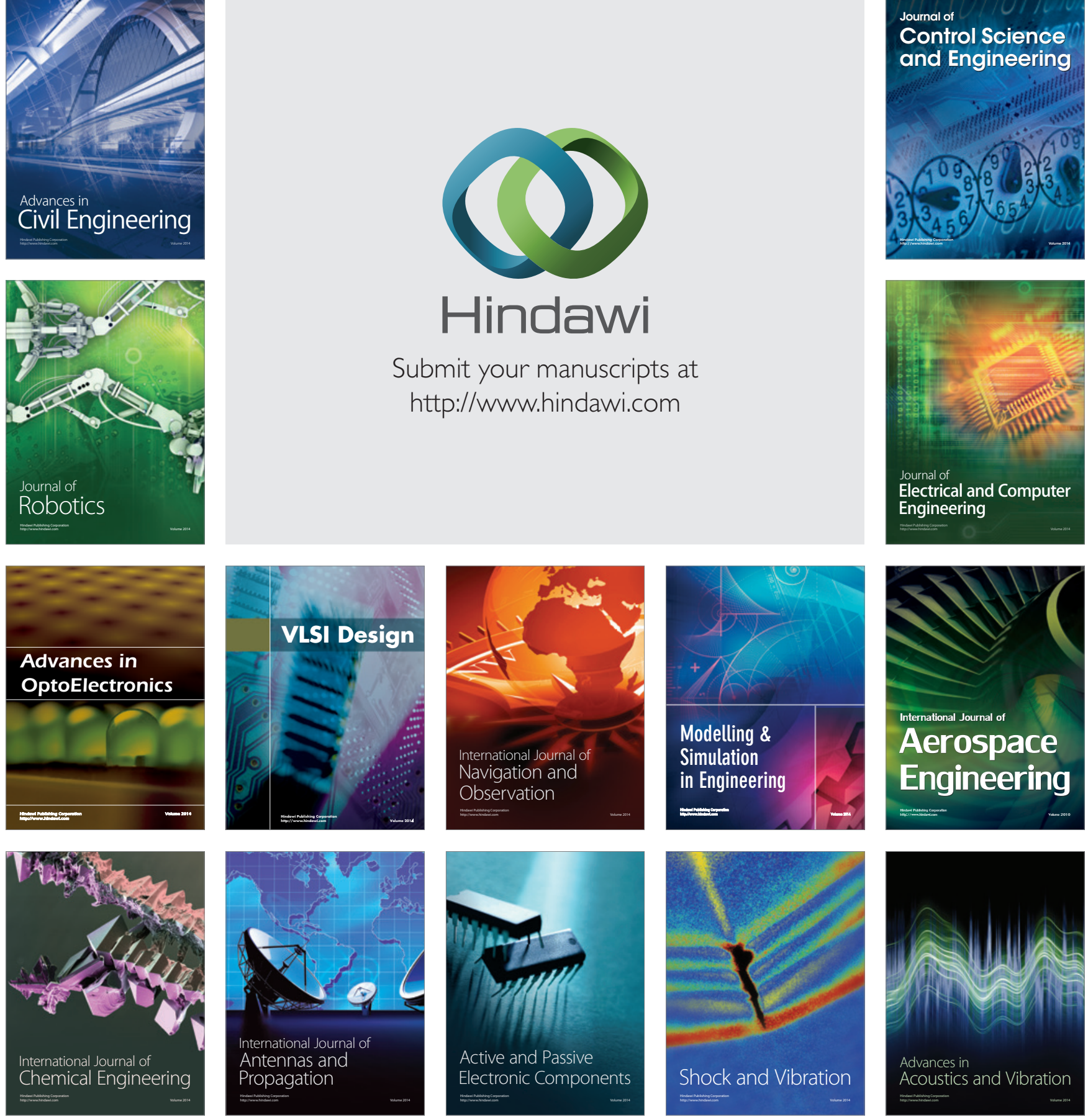